\title{
Mathematical Problems in Emerging Manufacturing Systems Management
}

\author{
Taho Yang, ${ }^{1}$ Mu-Chen Chen, ${ }^{2}$ Felix T. S. Chan, ${ }^{3}$ Chiwoon Cho, ${ }^{4}$ and Vikas Kumar ${ }^{5}$ \\ ${ }^{1}$ Institute of Manufacturing Information and Systems, National Cheng Kung University, Tainan 70101, Taiwan \\ ${ }^{2}$ Department of Transportation and Logistics Management, National Chiao Tung University, Taipei 100, Taiwan \\ ${ }^{3}$ Department of Industrial and Systems Engineering, The Hong Kong Polytechnic University, Hong Kong \\ ${ }^{4}$ Department of Industrial Engineering, University of Ulsan, Ulsan 680-749, Republic of Korea \\ ${ }^{5}$ Bristol Business School, University of the West of England, Bristol BS16 1QY, UK
}

Correspondence should be addressed to Taho Yang; tyang@mail.ncku.edu.tw

Received 8 April 2015; Accepted 8 April 2015

Copyright (C) 2015 Taho Yang et al. This is an open access article distributed under the Creative Commons Attribution License, which permits unrestricted use, distribution, and reproduction in any medium, provided the original work is properly cited.

This special issue aims to address the mathematical problems associated with the management of innovative emerging manufacturing systems. The scope of innovative manufacturing systems management in this special issue addresses the emerging issues from production and operations management, manufacturing strategy, lean/agile manufacturing, supply chain and logistics management, healthcare systems management, and so forth. The contributions gathered in this special issue offer a snapshot of different interesting researches, problems, and solutions. In the following, we briefly highlight these topics and synthesize the content of each paper.

The paper "Impacts of Transportation Cost on Distribution-Free Newsboy Problems," by M.-H. Shu et al., addresses a distribution-free newsboy problem (DFNP) for a vendor to decide a product's stock quantity in a single-period inventory system to sustain its least maximumexpected profits. The transportation cost is formulated as a function of shipping quantity and is modeled as a nonlinear regression form. An optimal solution of the order quantity is computed on the basis of Newton's approach to ameliorate its complexity of computation. The empirical results are quite competitive with the results from the existing literature.

The paper "The Integration of Group Technology and Simulation Optimization to Solve the Flow Shop with Highly Variable Cycle Time Process: A Surgery Scheduling Case Study," by T. K. Wang et al., introduces a case of healthcare system application. It proposes an algorithm that allows the estimation of the mean effective process time and the coefficient of variation. It also develops a group technology based takt time. A simulation model is combined with the case study, and the capacity buffers are optimized against the remaining variability for each group. The empirical results from a practical application are quite promising.

The paper "Undesirable Outputs' Presence in Centralized Resource Allocation Model," by G. Tohidi et al., extends the existing Data Envelopment Analysis (DEA) literature and proposes a new Centralized Resource Allocation (CRA) model to assess the overall efficiency of system consisting of Decision Making Units (DMUs) by using directional distance function when DMUs produce desirable and undesirable outputs.

The paper "A Multiple Attribute Group Decision Making Approach for Solving Problems with the Assessment of Preference Relations," by T. Yang et al., proposes to use a fuzzy preference relations matrix which satisfies additive consistency in solving a multiple attribute group decision making (MAGDM) problem. It takes a heterogeneous group of experts into consideration. A numerical example is used to test the proposed approach; and the results illustrate that the method is simple, effective, and practical.

The paper "Integrated Supply Chain Cooperative Inventory Model with Payment Period Being Dependent on Purchasing Price under Defective Rate Condition," by M.-F. Yang 
et al., aims at finding the maximum of the joint expected total profit and at coming up with a suitable inventory policy. It solves the trade-off between increased postponed payment deadline and the decreased profit for a buyer and vice versa for a vendor. Its numerical illustrations provide useful managerial insights.

The paper "Clustering Ensemble for Identifying Defective Wafer Bin Map in Semiconductor Manufacturing," by C.-Y. $\mathrm{Hsu}$, proposes a clustering ensemble approach to facilitate wafer bin map defect detection problem from semiconductor manufacturing. It adopts a series of algorithms to solve the proposed problem such as mountain function, $k$-means, particle swarm optimization, and neural network model. The numerical results are promising.

The paper "Joint Optimization Approach of Maintenance and Production Planning for a Multiple-Product Manufacturing System," by L. Mifdal et al. deals with the problem of maintenance and production planning for randomly failing multiple-product manufacturing system. It establishes sequentially an economical production plan and an optimal maintenance strategy, taking into account the influence of the production rate on the system's degradation. Analytical models are developed in order to minimize sequentially the total production/inventory cost and then the total maintenance cost. Finally, a numerical example is presented to illustrate the usefulness of the proposed approach.

The paper "The Dynamics of Bertrand Model with Technological Innovation," by F. Wang et al., studied the dynamics of a Bertrand duopoly game with technology innovation, which contains bounded rational and naive players. The stability of the equilibrium point, the bifurcation, and chaotic behavior of the dynamic system have been analyzed. It concludes that technology innovation can enlarge the stability region of the speed and control the chaos of the dynamic system effectively.

\section{Acknowledgments}

The guest editors would like to deeply thank all the authors, the reviewers, and the Editorial Board involved in the preparation of this issue.

Taho Yang

Mu-Chen Chen

Felix T. S. Chan

Chiwoon Cho

Vikas Kumar 


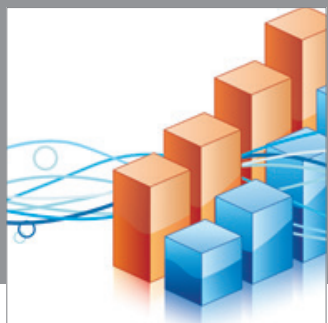

Advances in

Operations Research

mansans

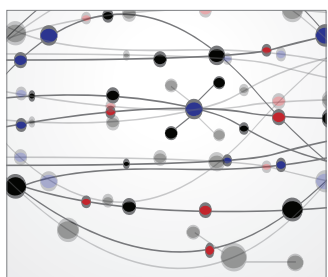

The Scientific World Journal
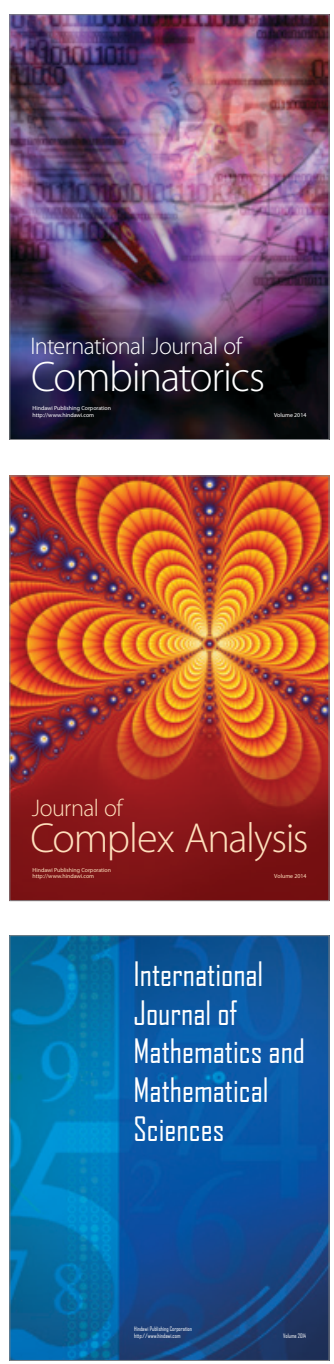
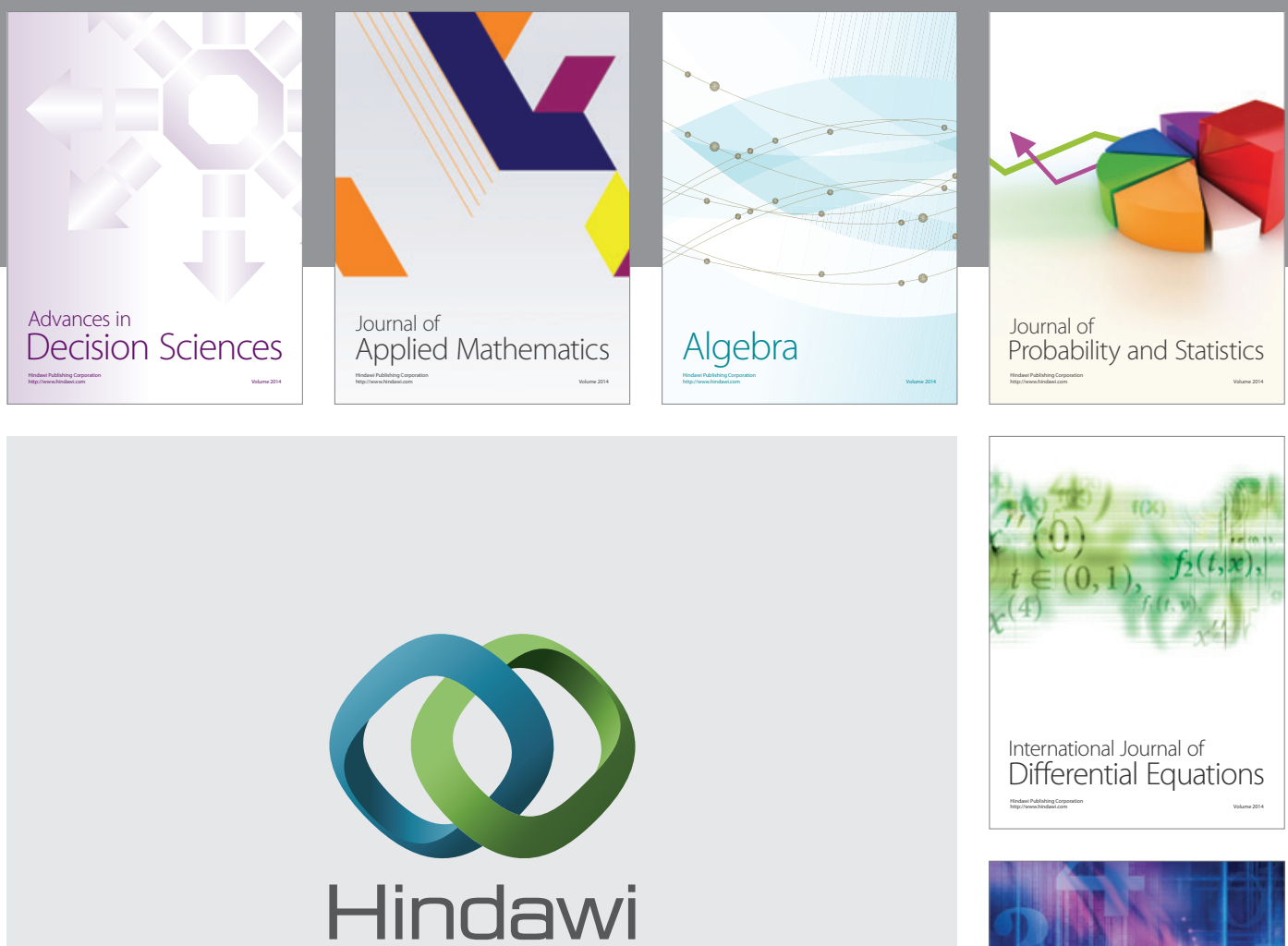

Submit your manuscripts at http://www.hindawi.com
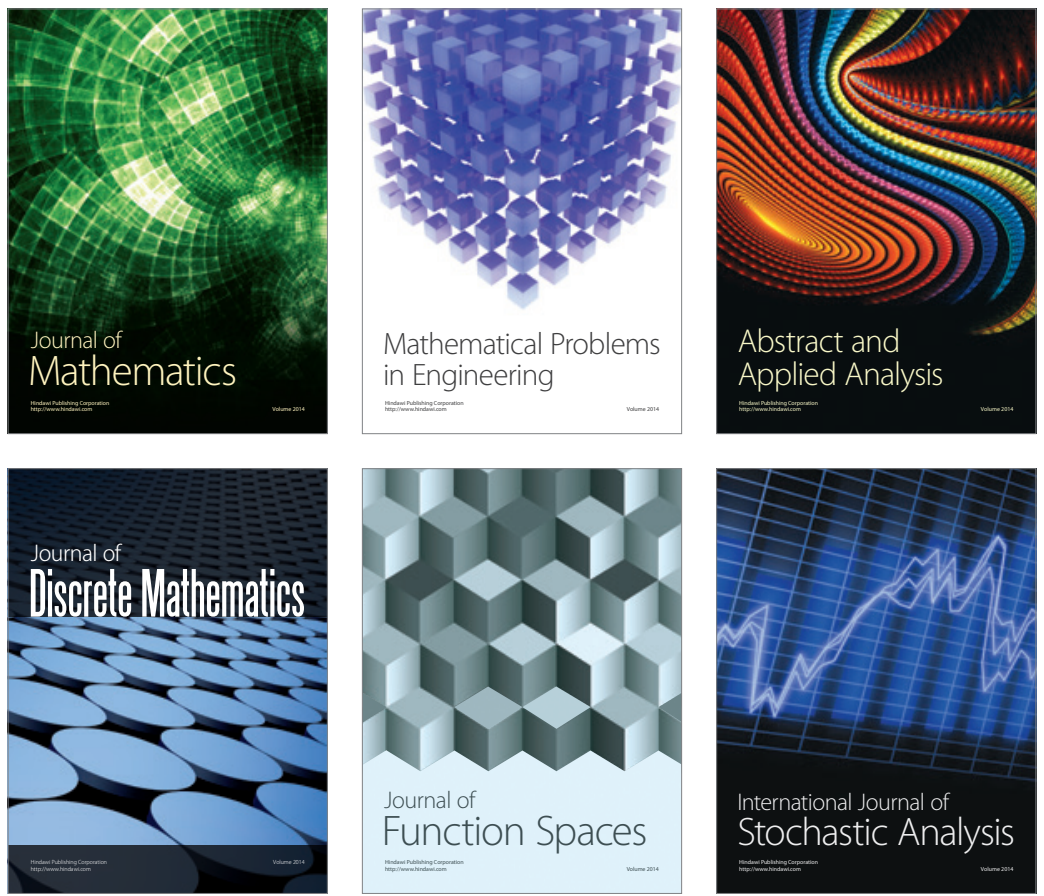

Journal of

Function Spaces

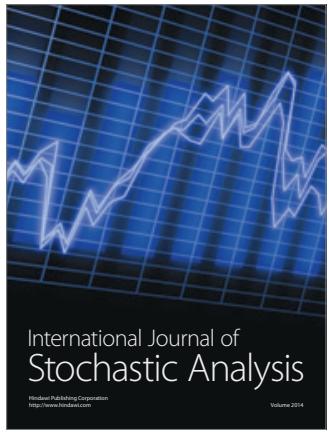

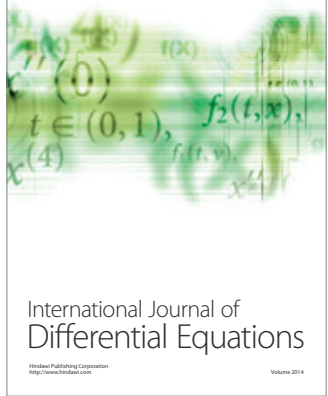
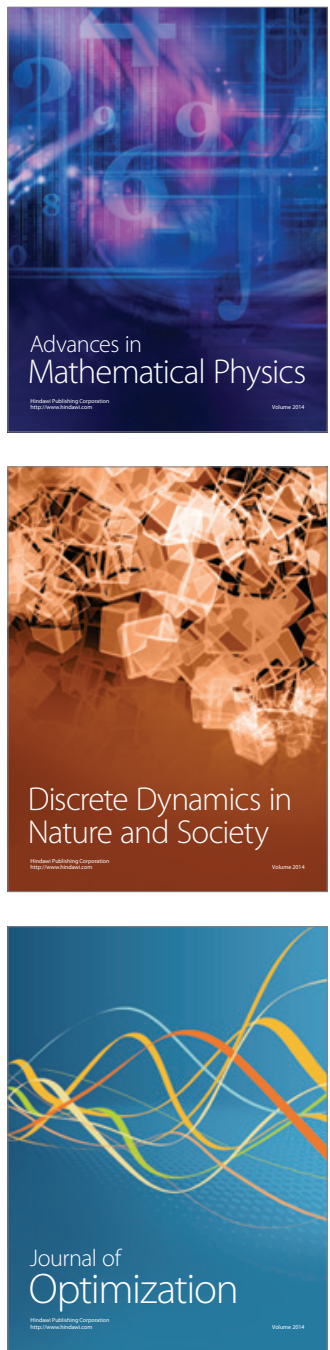